\title{
Stabilization of Measurement-Integrated Simulation by Elucidation of Destabilizing Mechanism*
}

\author{
Toshiyuki HAYASE**, Kentaro IMAGAWA**, \\ Kenichi FUNAMOTO** and Atsushi SHIRAI** \\ **Institute of Fluid Science, Tohoku University, \\ 2-1-1 Katahira, Aoba-ku, Sendai, 980-8577, Japan \\ E-mail: hayase@ifs.tohoku.ac.jp
}

\begin{abstract}
Measurement-integrated (MI) simulation is a numerical flow analysis method with a feedback mechanism from measurement of a real flow. It correctly reproduces a real flow under inherent ambiguity in a mathematical model or a computational condition. In this paper we theoretically investigated the destabilization phenomenon of MI simulation, in which analysis error suddenly increases at some critical feedback gain. This phenomenon has been considered as instability of a closed-loop feedback system, but present study treated it as that of a numerical scheme. First, the mechanism of the destabilization phenomenon was investigated based on the sufficient condition of the convergence of iterative calculation of existing MI simulation. It was found that the feedback signal in the source term destabilized the iterative calculation. Then, a new MI simulation scheme was derived by evaluating the feedback signal in the linear term to remove the cause of the destabilization. The validity of the present theoretical analysis was verified for examples treated in former studies of MI simulations: blood flow in an aneurismal aorta with ultrasonic measurement, blood flow in a cerebral aneurism with magnetic resonance measurement, Karman vortex street behind a square cylinder with PIV measurement, and fully developed turbulent flow in a square duct with ideal measurement. Occurrences of destabilization phenomenon in all the examples were well explained by the condition of this study, especially for cases of relatively small time steps and large feedback gains. Furthermore, the new MI simulation scheme realized the analysis without the destabilization phenomenon. The present theoretical result confirming that the destabilization phenomenon is not the instability of the feedback system but that of a numerical scheme is generally applicable to MI simulations using the velocity error for the feedback signal.
\end{abstract}

Key words: Measurement-Integrated Simulation, Destabilization Phenomenon, Theoretical Analysis, Critical Feedback Gain, Stabilized Scheme

\section{Introduction}

Obtaining accurate and detailed information of real flows is a critical issue in many fields such as weather forecasting, flight control, intraoperative blood flow monitoring, and nuclear power plant operation. Measurement and numerical simulation are widely used for analysis of flow phenomena. Generally speaking, measurement is the most direct technique to obtain the state of real flow within a range of its accuracy, but it is difficult to measure the complete states of flow, such as pressure and velocity, which widely spread in space and

Received 7 Sep., 2010 (No. 10-0398)

DOI: 10.1299/jst.5.632]

Copyright $\odot 2010$ by JSME 
time. On the other hand, by use of numerical simulation, the complete flow states can be obtained, but it is essentially difficult to exactly reproduce the real flow due to inevitable disturbances or ambiguity in initial or boundary conditions.

In order to overcome the difficulty in exactly reproducing real flows, various methods have been proposed to integrate measurement and simulation. In the meteorological field, many studies have been performed for data assimilation to obtain initial conditions for numerical weather prediction. Recently, the four-dimensional variational method (4D VAR) and the ensemble Kalman filter are being intensively studied ${ }^{(1)(2)}$. The Tichonov regularization technique, which is common in inverse problems, has been applied to estimate the pressure distribution around an airfoil in the aerospace field ${ }^{(3)}$. In the field of visualization measurement, pressure and velocity distributions are obtained by integrating PIV measurement and flow simulation ${ }^{(4)}$. State estimation methods in control theory, such as the observer or the Kalman filter, have been applied to flow problems. Unsteady flow in a pipe has been analyzed with the Kalman filter ${ }^{(5)}$, and measurement-integrated simulation (hereafter abbreviated as "MI simulation") has been proposed by applying the concept of the observer to a flow simulation ${ }^{(6)(7)}$.

In MI simulation, a time-dependent flow simulation is performed by applying a feedback signal which is proportional to the difference between the simulation result and the measurement of a real flow (see Fig. 1). In practice, modification of the computational scheme is quite simple, only external force terms being added to the governing equations corresponding to the feedback signal. If the feedback law is designed appropriately, the computational result asymptotically converges to the state of the real flow by the effect of continuous correction with measurement data, and the result of MI simulation can be used as a good estimation of the real flow after some convergence time. This principle of MI simulation is the same as that of the observer in control theory, and the characteristic point of MI simulation is that a large dimensional nonlinear model of computational fluid dynamics (CFD) is used as a mathematical model. This technique has been successfully applied to a variety of flow problems: a fully developed turbulent flow in a square duct ${ }^{(6)(7)}$, Karman vortex street behind a square cylinder in a wind tunnel with pressure sensor measurement on a cylinder surface or velocity field measurement by PIV $^{(8)(9)}$, blood flow in an aortic aneurysm with Doppler velocity measurement using an ultrasonic diagnosis device $^{(10)(11)}$, blood flow in an aneurism at a cerebral artery bifurcation with velocity measurement using MRI diagnosis equipment ${ }^{(12)}$, and turbulent flow through an orifice with pressure measurement ${ }^{(13)}$.

In designing MI simulation, the general theory of observers cannot be directly applied since the basic equations of MI simulation are nonlinear and do not have minimal

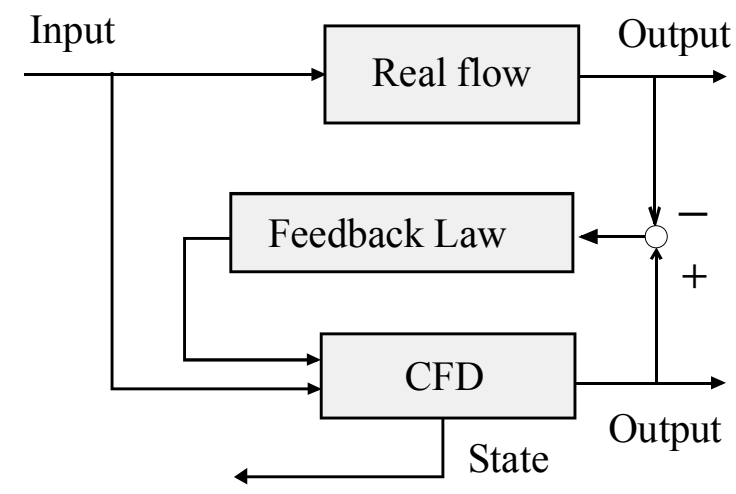

Fig. 1 Block diagram of measurement-integrated simulation 
dimensions. Recently, a theoretical study to design MI simulation based on the linearized error dynamics appeared, but its application is limited to simple problems ${ }^{(14)}$. Therefore, design of the feedback law in existing MI simulations is made by trial and error based on physical considerations.

A problem in the MI simulation design is the occurrence of the destabilization phenomenon, in which the analysis error suddenly increases above some critical value of the feedback gain. In a previous study of ultrasonic-measurement-integrated simulation of blood flow in an aneurismal aorta, it was pointed out that the critical feedback gain was inversely proportional to the time step of the simulation ${ }^{(11)}$, but neither the generality of this relation nor the mechanism of the destabilization phenomenon has been clarified. In control theory it is well known that a closed-loop system with a simple proportional feedback mostly undergoes instability with increasing feedback gain ${ }^{(15)}$. Since MI simulation is a typical closed-loop feedback system, the destabilization phenomenon has naturally been considered to be the instability of the feedback system ${ }^{(6)(7)}$. Another possible cause of the instability of a numerical scheme has not been considered. The steady error and the time constant are important parameters determining the reproducibility of real flows and traceability to unsteady flows, respectively, and they decrease with increasing the feedback gain in ordinary situations. Therefore, the existence of the upper limit in the feedback gain due to the destabilization phenomenon is a serious problem in MI simulation design. In order to solve this problem, it is critically important to elucidate the mechanism of the destabilization phenomenon and to establish a new MI simulation scheme stabilized by removing the cause of the destabilization.

This paper is a theoretical study to elucidate the mechanism of the destabilization phenomenon and to establish a new stabilized MI simulation scheme. Especially we focus on the instability of numerical scheme instead of that of the feedback system as a possible cause of the destabilization. In section 2 , the mechanism of the destabilization phenomenon is theoretically investigated based on the fundamental equations of MI simulation, and a new MI simulation scheme is then derived. Validity of the theoretical analysis is verified in section 3 for various examples: ultrasonic-measurement-integrated simulation of blood flow in an aneurismal aorta, MR-measurement-integrated simulation of blood flow in a cerebral aneurism, PIV-measurement-integrated simulation of Karman vortex street behind a square cylinder, and general MI simulation of a fully developed turbulent flow in a square duct. Conclusions of this work are summarized in section 4 .

\section{Nomenclature}
A : general matrix
f : $\quad$ external force, body force divided by fluid density
$\mathbf{f}_{N}$ : discretized external force (feedback signal), $3 \mathrm{~N}$ dimensional vector
$\mathbf{g}_{N}$ : discretization of convection and diffusion terms of Navier-Stokes equation, $3 \mathrm{~N}$ dimensional vector function of $\mathbf{u}_{N}$
$\mathbf{G}$ : linearization of nonlinear function $\mathbf{g}_{N}, 3 \mathrm{~N} \times 3 \mathrm{~N}$ matrix
$\tilde{\mathbf{G}}$ : coefficients subtracted from $\mathbf{G}, 3 \mathrm{~N} \times 3 \mathrm{~N}$ matrix
$\mathbf{K}$ : feedback gain matrix, $3 \mathrm{~N} \times 3 \mathrm{~N}$ matrix
$n$ : index of iterative calculation
$N$ : number of computational grid points
$p$ : $\quad$ pressure field divided by fluid density
$\mathbf{p}_{N}$ : discretized pressure field divided by fluid density, $\mathrm{N}$ dimensional vector dimensional vector function of $\mathbf{u}_{N}$
$t$ : time
$\mathbf{q}_{N}$ : discretization of divergence of convection term in pressure equation, $\mathrm{N}$ 
u : velocity vector field

$\mathbf{u}_{N}$ : discretized velocity vector field, $3 \mathrm{~N}$ dimensional vector

$\mathbf{x}$ : general vector

$\alpha, \beta$ : dividing points of $\mathbf{u}_{N}^{(n-1)}$ and $\mathbf{u}_{N}^{(n-2)}, 3 \mathrm{~N}$ dimensional vector

$\Delta t$ : time step

$\boldsymbol{\Phi}\left(\mathbf{u}_{N}^{(n-1)}\right)$ : Coefficients of linearized term evaluated with latest values in iterative calculation, $3 \mathrm{~N} \times 3 \mathrm{~N}$ matrix

$\boldsymbol{\Gamma}\left(\mathbf{u}_{N}^{(n-1)}\right)$ : term evaluated with previous values in iterative calculation, $3 \mathrm{~N}$ dimensional vector

$v$ : kinematic viscosity

$\nabla_{N}$ : discretization of gradient operator $\nabla, 3 \mathrm{~N} \times \mathrm{N}$ dimensional matrix

$\Delta_{N}$ : discretization of Laplace operator $\Delta, \mathrm{N} \times \mathrm{N}$ dimensional matrix

Superscripts

(n): values at $n$-th iteration

*: values of real flows

': derivative with respect to $\mathbf{u}_{N}$

Subscripts

$\mathrm{N}$ : discretized values

$\mathrm{N},(-1)$ : the first component "N" represents discretized values and the second one "(-1)" represents values at previous time step

crit: critical values for destabilization phenomenon

\section{Theoretical Analysis of Destabilization Phenomenon}

In this section, the mechanism of destabilization phenomenon is theoretically investigated based on the fundamental equations of MI simulation, and then a stabilized MI simulation scheme is derived by removing the cause of the destabilization.

\subsection{Destabilization Mechanism}

Governing equations of MI simulation are the Navier-Stokes equation including an external force term and the pressure equation.

$$
\begin{aligned}
& \frac{\partial \mathbf{u}}{\partial t}=-(\mathbf{u} \cdot \nabla) \mathbf{u}+v \Delta \mathbf{u}-\nabla p+\mathbf{f}, \\
& \Delta p=-\nabla \cdot((\mathbf{u} \cdot \nabla) \mathbf{u})+\nabla \cdot \mathbf{f},
\end{aligned}
$$

where $p$ is the pressure divided by the fluid density and $\mathbf{f}$ is the external force, or the body force divided by the fluid density, which is used as the feedback signal.

After discretization in space coordinates we obtain the following equations:

$$
\begin{aligned}
& \frac{d \mathbf{u}_{N}}{d t}=\mathbf{g}_{N}\left(\mathbf{u}_{N}\right)-\nabla_{N} \mathbf{p}_{N}+\mathbf{f}_{N}, \\
& \Delta_{N} \mathbf{p}_{N}=\mathbf{q}_{N}\left(\mathbf{u}_{N}\right)+\nabla_{N}{ }^{T} \mathbf{f}_{N},
\end{aligned}
$$

where $\mathbf{u}_{N}$ denotes the $3 \mathrm{~N}$ dimensional vector consisting of velocity vectors at the computational grid points (N: number of grid points), $\mathbf{p}_{N}$ is the $\mathrm{N}$ dimensional vector of the pressure divided by the density, $\mathbf{g}_{N}$ and $\mathbf{q}_{N}$ are the $3 \mathrm{~N}$ and $\mathrm{N}$ dimensional nonlinear functions of $\mathbf{u}_{N}$ derived from the first and the second terms of Eq. (1) and the first term of Eq. (2) in the right-hand side, respectively, $\nabla_{N}$ and $\Delta_{N}$ are the $3 N \times N$ and $N \times N$ dimensional matrices of discretized form of the gradient $\nabla$ and Laplace operator $\Delta$, respectively. In this paper we consider the case in which the $3 \mathrm{~N}$ dimensional vector of the 
external force term (feedback signal), $\mathbf{f}_{N}$, is given as a linear function of the velocity error vector:

$$
\mathbf{f}_{N}=-\mathbf{K}\left(\mathbf{u}_{N}-\mathbf{u}_{N}^{*}\right),
$$

where $\mathbf{K}$ is the $3 \mathrm{~N} \times 3 \mathrm{~N}$ dimensional feedback gain matrix, and $\mathbf{u}_{N}^{*}$ is the $3 \mathrm{~N}$ dimensional vector of measured velocity vectors. It is noted that unmeasurable elements of $\mathbf{u}_{N}^{*}$ and corresponding columns of $\mathbf{K}$ are set to zero.

\section{First order implicit scheme}

First, we consider the case in which the time derivative term of Eq. (3) is discretized with the first order implicit scheme. In this case, considering Eq. (5), we obtain the following expression.

$$
\frac{\mathbf{u}_{N}-\mathbf{u}_{N,(-1)}}{\Delta t}=\mathbf{g}_{N}\left(\mathbf{u}_{N}\right)-\nabla_{N} \mathbf{p}_{N}-\mathbf{K}\left(\mathbf{u}_{N}-\mathbf{u}_{N}^{*}\right),
$$

where $(-1)$ of the second component of subscript of left-hand side represents the value of the former time step.

In the present study we assume the SIMPLER method ${ }^{(16)}$ as the numerical scheme. Because of the space limitation, the standard way to deal with the pressure equation and the pressure correction equation is omitted in the followings. Detailed description of the numerical scheme is given in the reference ${ }^{(16)}$.

Since the first term of the right-hand side of Eq. (6) is nonlinear with respect to $\mathbf{u}_{N}$, the term is linearized and the resultant linear equation is repeatedly solved until the convergent solution is obtained. From Eq. (6) the fundamental equation for the iterative calculation of MI simulation in former studies is derived as Eq. (7) with the initial condition, $\mathbf{u}_{N}^{(0)}$.

$$
\begin{aligned}
\boldsymbol{\Phi}\left(\mathbf{u}_{N}^{(n-1)}\right) \mathbf{u}_{N}^{(n)} & =\boldsymbol{\Gamma}\left(\mathbf{u}_{N}^{(n-1)}\right), \quad n=1,2,3 \cdots \\
\boldsymbol{\Phi}\left(\mathbf{u}_{N}^{(n-1)}\right) & =\frac{\mathbf{I}}{\Delta t}-\mathbf{G}\left(\mathbf{u}_{N}^{(n-1)}\right)+\tilde{\mathbf{G}}\left(\mathbf{u}_{N}^{(n-1)}\right) \\
\boldsymbol{\Gamma}\left(\mathbf{u}_{N}^{(n-1)}\right) & =\frac{\mathbf{u}_{N,(-1)}}{\Delta t}+\tilde{\mathbf{G}}\left(\mathbf{u}_{N}^{(n-1)}\right) \mathbf{u}_{N}^{(n-1)}-\nabla_{N} \mathbf{p}_{N}\left(\mathbf{u}_{N}^{(n-1)}\right)-\mathbf{K} \mathbf{u}_{N}^{(n-1)}+\mathbf{K} \mathbf{u}_{N}^{*} .
\end{aligned}
$$

with

$$
\mathbf{G}\left(\mathbf{u}_{N}^{(n-1)}\right)=\left.\frac{d \mathbf{g}_{N}}{d \mathbf{u}_{N}}\right|_{\mathbf{u}_{N}^{(n-1)}}
$$

The above linear equation for $\mathbf{u}_{N}^{(n)}$ is solved with given $\mathbf{u}_{N}^{(n-1)}$ where $n$ in the superscript is the index of iteration. A $\mathbf{G}\left(\mathbf{u}_{N}^{(n-1)}\right)$ is the $3 \mathrm{~N} \times 3 \mathrm{~N}$ matrix of linearized coefficients obtained from the nonlinear term $\mathbf{g}_{N}$ in Eq. (6) by evaluating its coefficients with the values at the former iteration step $\mathbf{u}_{N}^{(n-1)}$. The matrix $\tilde{\mathbf{G}}\left(\mathbf{u}_{N}^{(n-1)}\right)$ represents the terms removed from those with the matrix $\mathbf{G}\left(\mathbf{u}_{N}^{(n-1)}\right)$ in $\Phi$, and added in $\Gamma$ to keep the consistency. Example of $\tilde{\mathbf{G}}\left(\mathbf{u}_{N}^{(n-1)}\right)$ is found in the common treatment of the discretized convection terms in which some terms are moved to the source term in order to improve convergence property of the numerical scheme ${ }^{(17)}$. The $\mathbf{p}_{N}\left(\mathbf{u}_{N}^{(n-1)}\right)$ is obtained from the discretized pressure equation derived from Eq. (4). It is noted that the feedback signal is included in $\Gamma$ as the source terms in the formulation of MI simulation of former studies.

Taking the difference between Eq. (7) evaluated at iteration number $n$ and that evaluated at $n-1$, we obtain the following expression:

$$
\boldsymbol{\Phi}\left(\mathbf{u}_{N}^{(n-1)}\right) \mathbf{u}_{N}^{(n)}-\boldsymbol{\Phi}\left(\mathbf{u}_{N}^{(n-2)}\right) \mathbf{u}_{N}^{(n-1)}=\boldsymbol{\Gamma}\left(\mathbf{u}_{N}^{(n-1)}\right)-\boldsymbol{\Gamma}\left(\mathbf{u}_{N}^{(n-2)}\right)
$$

Applying the mean value theorem, the left-hand side of the above equation is written as:

$$
\begin{aligned}
& \boldsymbol{\Phi}\left(\mathbf{u}_{N}^{(n-1)}\right) \mathbf{u}_{N}^{(n)}-\boldsymbol{\Phi}\left(\mathbf{u}_{N}^{(n-1)}\right) \mathbf{u}_{N}^{(n-1)}+\boldsymbol{\Phi}\left(\mathbf{u}_{N}^{(n-1)}\right) \mathbf{u}_{N}^{(n-1)}-\boldsymbol{\Phi}\left(\mathbf{u}_{N}^{(n-2)}\right) \mathbf{u}_{N}^{(n-1)}, \\
& =\boldsymbol{\Phi}\left(\mathbf{u}_{N}^{(n-1)}\right)\left(\mathbf{u}_{N}^{(n)}-\mathbf{u}_{N}^{(n-1)}\right)+\boldsymbol{\Phi}^{\prime}(\boldsymbol{\alpha}) \mathbf{u}_{N}^{(n-1)}\left(\mathbf{u}_{N}^{(n-1)}-\mathbf{u}_{N}^{(n-2)}\right),
\end{aligned}
$$


where $\boldsymbol{\Phi}^{\prime}$ is a derivative of $\Phi$ with respect to $\mathbf{u}_{N}$, and $\alpha$ is an appropriate dividing point of $\mathbf{u}_{N}^{(n-1)}$ and $\mathbf{u}_{N}^{(n-2)}$. Introducing the above relation into the left-hand side of Eq. (8), we obtain the following relation:

$$
\boldsymbol{\Phi}\left(\mathbf{u}_{N}^{(n-1)}\right)\left(\mathbf{u}_{N}^{(n)}-\mathbf{u}_{N}^{(n-1)}\right)=\left(-\boldsymbol{\Phi}^{\prime}(\boldsymbol{\alpha}) \mathbf{u}_{N}^{(n-1)}+\boldsymbol{\Gamma}^{\prime}(\boldsymbol{\beta})\right)\left(\mathbf{u}_{N}^{(n-1)}-\mathbf{u}_{N}^{(n-2)}\right),
$$

where $\Gamma^{\prime}$ is a derivative of $\Gamma$ with respect to $\mathbf{u}_{N}$, and $\beta$ is an appropriate dividing point of $\mathbf{u}_{N}^{(n-1)}$ and $\mathbf{u}_{N}^{(n-2)}$.

Taking the norms of Eq. (10), the following relation generally applies:

$$
\left\|\mathbf{u}_{N}^{(n)}-\mathbf{u}_{N}^{(n-1)}\right\| \leq \frac{\left\|-\boldsymbol{\Phi}^{\prime}(\boldsymbol{\alpha}) \mathbf{u}_{N}^{(n-1)}+\boldsymbol{\Gamma}^{\prime}(\boldsymbol{\beta})\right\|}{\left\|\boldsymbol{\Phi}\left(\mathbf{u}_{N}^{(n-1)}\right)\right\|}\left\|\mathbf{u}_{N}^{(n-1)}-\mathbf{u}_{N}^{(n-2)}\right\| .
$$

Norms of matrices in the above expression are defined as the induced norms which represent the maximum magnification of linear transformation as ${ }^{(18)}$

$$
\|\mathbf{A}\|=\max _{\mathbf{x} \neq \mathbf{0}} \frac{\|\mathbf{A} \mathbf{x}\|}{\|\mathbf{x}\|} .
$$

From Eq. (11), the sufficient condition of convergence of $\mathbf{u}_{N}^{(n)}$ as $n \rightarrow \infty$ is derived so that the coefficient in the iteration is less than 1 , or

$$
\frac{\left\|-\boldsymbol{\Phi}^{\prime}(\boldsymbol{\alpha}) \mathbf{u}_{N}^{(n-1)}+\boldsymbol{\Gamma}^{\prime}(\boldsymbol{\beta})\right\|}{\left\|\boldsymbol{\Phi}\left(\mathbf{u}_{N}^{(n-1)}\right)\right\|}<1 .
$$

Substituting the second and third expressions of Eqs. (7) into the above expression, we obtain the following relation:

$$
\frac{\left\|\mathbf{G}^{\prime}(\boldsymbol{\alpha}) \mathbf{u}_{N}^{(n-1)}-\tilde{\mathbf{G}}^{\prime}(\boldsymbol{\alpha}) \mathbf{u}_{N}^{(n-1)}+\tilde{\mathbf{G}}^{\prime}(\boldsymbol{\beta}) \boldsymbol{\beta}+\tilde{\mathbf{G}}(\boldsymbol{\beta})-\nabla_{N} \mathbf{p}_{N}^{\prime}(\boldsymbol{\beta})-\mathbf{K}\right\|}{\left\|\frac{\mathbf{I}}{\Delta t}-\mathbf{G}\left(\mathbf{u}_{N}^{(n-1)}\right)+\tilde{\mathbf{G}}\left(\mathbf{u}_{N}^{(n-1)}\right)\right\|}<1,
$$

where $\mathbf{G}^{\prime}, \tilde{\mathbf{G}}^{\prime}$, and $\mathbf{p}_{N}^{\prime}$ are derivatives of $\mathbf{G}, \tilde{\mathbf{G}}$, and $\mathbf{p}_{N}$ with respect to $\mathbf{u}_{N}$.

We consider the case in which the computational time step $\Delta t$ is relatively small and the feedback gain is relatively large. The leading term of the numerator of Eq. (14) is then considered to be $-\mathbf{K}$ and that of the denominator be $\mathbf{I} / \Delta \mathrm{t}$, and therefore we obtain an approximate formulation for the sufficient condition of convergence for the iterative calculation of MI simulation as:

$$
\|\mathbf{K}\|<\frac{1}{\Delta t}
$$

and the critical value of the feedback gain matrix norm above which the destabilization phenomenon occurs as:

$$
\|\mathbf{K}\|_{\text {crit }}=\frac{1}{\Delta t}
$$

This relation agrees with the empirical relation obtained in a former study ${ }^{(11)}$.

By summarizing the above discussion, the destabilization phenomenon of existing MI simulation is ascribed to the fact that a large feedback signal in the source term determines the leading term of the numerator of the coefficient for the successive change in the iterative calculation, and, therefore, divergence occurs with the coefficient larger than 1 , or the feedback gain larger than the critical value.

Patankar gave four rules which ensure the stable convergence of a finite-volume-based algorithm towards a physically realistic numerical solution ${ }^{(16)}$. In Rule 3: Negative-slope linearization of the source term, he pointed out that an appropriate linearization of the source term is a key to obtain convergent solution. The present destabilization phenomenon 
of MI simulation is considered to be the result of an inappropriate treatment of the source term.

\section{Second order implicit scheme}

Next, we consider the case in which the time derivative term of Eq. (3) is discretized with the second order implicit scheme. In the authors' former studies of MI simulation, the following expression was used for the second order discretization of the time derivative $\operatorname{term}^{(19)}$.

$$
\frac{3 \mathbf{u}_{N}-4 \mathbf{u}_{N,(-1)}+\mathbf{u}_{N,(-2)}}{2 \Delta t}=\frac{\mathbf{u}_{N}-\mathbf{u}_{N,(-1)}}{\Delta t}+\frac{\mathbf{u}_{N}-2 \mathbf{u}_{N,(-1)}+\mathbf{u}_{N,(-2)}}{2 \Delta t},
$$

where (-1) and (-2) of the second component of subscript represent the values before one time step and two time steps, respectively. The right-hand side of the expression consists of the first order discretization and the correction term.

Iterative calculation is represented in the same way as in the case of the first order implicit scheme in Eq. (7) with different expressions in $\Gamma$ denoted by underline as

$$
\begin{aligned}
\boldsymbol{\Phi}\left(\mathbf{u}_{N}^{(n-1)}\right) \mathbf{u}_{N}^{(n)} & =\boldsymbol{\Gamma}\left(\mathbf{u}_{N}^{(n-1)}\right), \quad n=1,2,3 \cdots \\
\boldsymbol{\Phi}\left(\mathbf{u}_{N}^{(n-1)}\right) & =\frac{\mathbf{I}}{\Delta t}-\mathbf{G}\left(\mathbf{u}_{N}^{(n-1)}\right)+\tilde{\mathbf{G}}\left(\mathbf{u}_{N}^{(n-1)}\right) \\
\boldsymbol{\Gamma}\left(\mathbf{u}_{N}^{(n-1)}\right)= & \frac{\mathbf{u}_{N,(-1)}}{\Delta t}-\frac{\mathbf{u}_{N}^{(n-1)}-2 \mathbf{u}_{N,(-1)}+\mathbf{u}_{N,(-2)}}{2 \Delta t}+\tilde{\mathbf{G}}\left(\mathbf{u}_{N}^{(n-1)}\right) \mathbf{u}_{N}^{(n-1)} \\
& -\nabla_{N} \mathbf{p}_{N}\left(\mathbf{u}_{N}^{(n-1)}\right)-\mathbf{K} \mathbf{u}_{N}^{(n-1)}+\mathbf{K} \mathbf{u}_{N}^{*}
\end{aligned}
$$

It is noted that the correction term in Eq. (17) is included in the source term $\Gamma$. Introducing the second and third expressions in Eqs. (18) to Eq. (13), we obtain the following relation.

$$
\frac{\left\|\mathbf{G}^{\prime}(\boldsymbol{\alpha}) \mathbf{u}_{N}^{(n-1)}-\tilde{\mathbf{G}}^{\prime}(\boldsymbol{\alpha}) \mathbf{u}_{N}^{(n-1)}-\frac{\mathbf{I}}{2 \Delta t}+\tilde{\mathbf{G}}^{\prime}(\boldsymbol{\beta}) \boldsymbol{\beta}+\tilde{\mathbf{G}}(\boldsymbol{\beta})-\nabla_{N} \mathbf{p}_{N}^{\prime}(\boldsymbol{\beta})-\mathbf{K}\right\|}{\left\|\frac{\mathbf{I}}{\Delta t}-\mathbf{G}\left(\mathbf{u}_{N}^{(n-1)}\right)+\tilde{\mathbf{G}}\left(\mathbf{u}_{N}^{(n-1)}\right)\right\|}<1 .
$$

As with the discussion of the first order implicit scheme, we consider the case in which the computational time step $\Delta t$ is relatively small and the feedback gain is relatively large. The leading term of the numerator of Eq. (19) is then considered to be $-\mathbf{I} /(2 \Delta t)-\mathbf{K}$ and that of the denominator be $\mathbf{I} / \Delta t$, and therefore we obtain the following relation for an approximate formulation for the sufficient condition of convergence of MI simulation:

$$
\left\|\frac{\mathbf{I}}{2 \Delta t}+\mathbf{K}\right\|<\frac{1}{\Delta t} .
$$

Considering that the feedback gain matrix $\mathbf{K}$ has only positive or zero eigenvalues since the feedback signal is applied to the direction to reduce the error in MI simulation and that the term $\mathbf{I} /(2 \Delta t)$ shifts the eigenvalues of $\mathbf{K}$ in the amount of $1 /(2 \Delta t)$, we obtain the following relation:

$$
\|\mathbf{K}\|<\frac{1}{2 \Delta t}
$$

and the critical value of the feedback gain matrix norm as:

$$
\|\mathbf{K}\|_{\text {crit }}=\frac{1}{2 \Delta t} \text {. }
$$

The mechanism of the destabilization phenomenon of MI simulation with the second 
order implicit scheme is essentially the same as that for the first order scheme. The feedback gain is included as a leading term in the numerator of the coefficient determining the successive change in the iterative calculation. By comparing Eq. (22) with Eq. (16), it is noted that the critical gain for the second order implicit scheme is half that of the first order one.

\subsection{Stabilized MI Simulation Scheme}

From the discussion of the former section, the scenario of the destabilization phenomenon of MI simulation is described as follows. Since the feedback signal is included in the source term, the feedback gain matrix is included in the numerator of the coefficient which determines the magnification rate of the variation in the iteration process. When the feedback gain is larger than the critical value, the coefficient becomes larger than one, and the iterative calculation diverges. Therefore, it is naturally expected that the cause of the destabilization phenomenon is eliminated by evaluating the feedback signal in the linear term in the iterative calculation.

\section{First order implicit scheme}

First we consider the case of the first order implicit scheme. If we move the feedback term relating to $\mathbf{u}_{N}$ from the source term in the right-hand side to the linear term in the left side of the first expression of Eq. (7), we obtain the new iterative calculation scheme as follows:

$$
\begin{aligned}
\boldsymbol{\Phi}\left(\mathbf{u}_{N}^{(n-1)}\right) \mathbf{u}_{N}^{(n)} & =\boldsymbol{\Gamma}\left(\mathbf{u}_{N}^{(n-1)}\right), \quad n=1,2,3 \cdots \\
\boldsymbol{\Phi}\left(\mathbf{u}_{N}^{(n-1)}\right) & =\frac{\mathbf{I}}{\Delta t}-\mathbf{G}\left(\mathbf{u}_{N}^{(n-1)}\right)+\tilde{\mathbf{G}}\left(\mathbf{u}_{N}^{(n-1)}\right)+\underline{\mathbf{K}} \\
\boldsymbol{\Gamma}\left(\mathbf{u}_{N}^{(n-1)}\right) & =\frac{\mathbf{u}_{N,(-1)}}{\Delta t}+\tilde{\mathbf{G}}\left(\mathbf{u}_{N}^{(n-1)}\right) \mathbf{u}_{N}^{(n-1)}-\nabla_{N} \mathbf{p}_{N}\left(\mathbf{u}_{N}^{(n-1)}\right)+\mathbf{K} \mathbf{u}_{N}^{*}
\end{aligned},
$$

where modification from the original scheme is underlined. Substituting the above expressions into Eq. (13), we obtain the following relation.

$$
\frac{\left\|\mathbf{G}^{\prime}(\boldsymbol{\alpha}) \mathbf{u}_{N}^{(n-1)}-\tilde{\mathbf{G}}^{\prime}(\boldsymbol{\alpha}) \mathbf{u}_{N}^{(n-1)}+\tilde{\mathbf{G}}^{\prime}(\boldsymbol{\beta}) \boldsymbol{\beta}+\tilde{\mathbf{G}}(\boldsymbol{\beta})-\nabla_{N} \mathbf{p}_{N}^{\prime}(\boldsymbol{\beta})\right\|}{\left\|\frac{\mathbf{I}}{\Delta t}-\mathbf{G}\left(\mathbf{u}_{N}^{(n-1)}\right)+\tilde{\mathbf{G}}\left(\mathbf{u}_{N}^{(n-1)}\right)+\mathbf{K}\right\|}<1 .
$$

The above relation is satisfied for null feedback gain matrix if the original simulation scheme is properly formulated. Furthermore, this relation is satisfied for the feedback gain matrix having only positive or zero eigenvalues. The last condition for the gain matrix is always satisfied for appropriate MI simulation.

In summary, Eq. (23) represents the new stabilized MI simulation scheme without the destabilization phenomenon. It is noted that the numerical scheme proposed here is an application of the standard procedure of linearization of source terms in numerical $\operatorname{method}^{(16)}$. The validity of the proposed scheme is confirmed in the next section.

\section{Second order implicit scheme}

Similarly to the above first order case, we obtain the new iterative calculation scheme by replacing the underlined feedback term relating to $\mathbf{u}_{N}$ from $\Gamma$ to $\Phi$ in Eq. (18) as follows:

$$
\begin{aligned}
\boldsymbol{\Phi}\left(\mathbf{u}_{N}^{(n-1)}\right) \mathbf{u}_{N}^{(n)} & =\boldsymbol{\Gamma}\left(\mathbf{u}_{N}^{(n-1)}\right), \quad n=1,2,3 \cdots \\
\boldsymbol{\Phi}\left(\mathbf{u}_{N}^{(n-1)}\right)= & \frac{\mathbf{I}}{\Delta t}-\mathbf{G}\left(\mathbf{u}_{N}^{(n-1)}\right)+\tilde{\mathbf{G}}\left(\mathbf{u}_{N}^{(n-1)}\right)+\underline{\mathbf{K}} \\
\boldsymbol{\Gamma}\left(\mathbf{u}_{N}^{(n-1)}\right)= & \frac{\mathbf{u}_{N,(-1)}}{\Delta t}-\frac{\mathbf{u}_{N}^{(n-1)}-2 \mathbf{u}_{N,(-1)}+\mathbf{u}_{N,(-2)}}{2 \Delta t}+\tilde{\mathbf{G}}\left(\mathbf{u}_{N}^{(n-1)}\right) \mathbf{u}_{N}^{(n-1)} \\
& -\nabla_{N} \mathbf{p}_{N}\left(\mathbf{u}_{N}^{(n-1)}\right)+\mathbf{K u}_{N}^{*}
\end{aligned}
$$


Substituting the above expressions into Eq. (13), we obtain the following relation.

$$
\frac{\left\|\mathbf{G}^{\prime}(\boldsymbol{\alpha}) \mathbf{u}_{N}^{(n-1)}-\tilde{\mathbf{G}}^{\prime}(\boldsymbol{\alpha}) \mathbf{u}_{N}^{(n-1)}-\frac{\mathbf{I}}{2 \Delta t}+\tilde{\mathbf{G}}^{\prime}(\boldsymbol{\beta}) \boldsymbol{\beta}+\tilde{\mathbf{G}}(\boldsymbol{\beta})-\nabla_{N} \mathbf{p}_{N}^{\prime}(\boldsymbol{\beta})\right\|}{\left\|\frac{\mathbf{I}}{\Delta t}-\mathbf{G}\left(\mathbf{u}_{N}^{(n-1)}\right)+\tilde{\mathbf{G}}\left(\mathbf{u}_{N}^{(n-1)}\right)+\mathbf{K}\right\|}<1 .
$$

For the same reason as the first order case, the above relation is always satisfied for an appropriately designed MI simulation.

In summary, Eq. (25) represents the new stabilized MI simulation scheme for the second order implicit scheme without the destabilization phenomenon. The numerical scheme proposed here is also an application of the standard procedure of linearization of source terms in numerical method ${ }^{(16)}$.

\section{Confirmation of Theoretical Results by Numerical Examples}

\subsection{Methods}

In this section, the validity of the theoretical analysis presented in the former section is investigated with various examples of MI simulation. Treated flows and analysis conditions are summarized in Fig. 2 and Table 1. These flows are those treated in the authors' former studies $^{(7)(9)(10)-(12)}$. Figure 2(a) is an aneurismal descending aorta in which ultrasonicmeasurement-integrated (UMI) blood flow simulation was performed. We deal with the case in which an esophageal ultrasonic probe of a medical ultrasonic diagnosis equipment is used. Doppler velocity or the blood velocity component along an ultrasonic beam measured with the color Doppler mode ${ }^{(20)}$ was fed back to the simulation at all grid points in the aneurism. The geometry of Fig. 2(a) was used for the analysis of a pulsated flow ${ }^{(10)}$, and the geometry shortened by cutting both upstream and downstream ends was used for the analysis of a steady flow ${ }^{(11)}$. The standard solution, which is a model of a real flow, was obtained by specifying both upstream and downstream velocity profiles, while MI simulation was performed with a uniform parallel flow at the upstream boundary and the free stream condition at the downstream boundary since the exact boundary condition is

Table 1 Analysis condition

\begin{tabular}{|c|c|c|c|c|c|c|c|}
\hline Cases & $1 \mathrm{a}$ & $1 b$ & $2 \mathrm{a}$ & $2 b$ & 3 & $4 a$ & $4 b$ \\
\hline MI simulation & \multicolumn{2}{|c|}{ UMI simulation } & \multicolumn{2}{|c|}{ MR-MI simulation } & $\begin{array}{l}\text { PIV-MI } \\
\text { simulation }\end{array}$ & \multicolumn{2}{|c|}{$\begin{array}{l}\text { general MI } \\
\text { simulation }\end{array}$} \\
\hline Measurement & \multicolumn{2}{|c|}{$\begin{array}{l}\text { Ultrasound } \\
\text { (color Doppler mode) }\end{array}$} & \multicolumn{2}{|c|}{$\begin{array}{l}\text { Magnetic resonance } \\
\text { (phase contrast mode) }\end{array}$} & PIV & \multicolumn{2}{|c|}{ Not specified } \\
\hline Subject & \multicolumn{2}{|c|}{$\begin{array}{l}\text { Blood flow in } \\
\text { aneurismal aorta }\end{array}$} & \multicolumn{2}{|c|}{$\begin{array}{l}\text { Blood flow in } \\
\text { cerebral artery }\end{array}$} & $\begin{array}{l}\text { Karman } \\
\text { vortex }\end{array}$ & \multicolumn{2}{|c|}{$\begin{array}{l}\text { Turbulent } \\
\text { flow in square } \\
\text { duct }\end{array}$} \\
\hline $\begin{array}{l}\text { Steadiness of } \\
\text { target flow }\end{array}$ & Steady & Unsteady & Steady & Unsteady & Unsteady & \multicolumn{2}{|c|}{ Unsteady } \\
\hline $\begin{array}{l}\text { Time } \\
\text { derivative } \\
\text { discretization }\end{array}$ & 1st order & 2 nd order & \multicolumn{2}{|c|}{1 st order } & 2nd order & $\begin{array}{c}1 \mathrm{st} \\
\text { order }\end{array}$ & $\begin{array}{l}\text { 2nd } \\
\text { order }\end{array}$ \\
\hline Grid points & $40 \times 34 \times 49$ & $43 \times 30 \times 91$ & \multicolumn{2}{|c|}{$30 \times 27 \times 26$} & $111 \times 21$ & \multicolumn{2}{|c|}{$80 \times 40 \times 40$} \\
\hline $\begin{array}{l}\text { Reynolds } \\
\text { number }\end{array}$ & 1,200 & $2,700(\max )$ & 230 & $420(\max )$ & 1,200 & \multicolumn{2}{|c|}{9,000} \\
\hline References & (11) & (10) & \multicolumn{2}{|c|}{ (12) } & (9) & \multicolumn{2}{|c|}{ (7) } \\
\hline
\end{tabular}


usually unknown in a realistic situation.

Figure 2 (b) is an aneurism developed at bifurcation of a cerebral artery in which steady and unsteady blood flow analysis were performed with MR-measurement-integrated simulation (MR-MI simulation) ${ }^{(12)}$. In this methodology velocity vector data measured by PC-MRI (Phase Contrast MRI) method are fed back to the simulation to obtain an accurate blood flow field under the condition of insufficient resolution in space and time or accuracy of common PC-MRI measurement. The boundary condition of the standard solution, or a model of a real flow, was determined by referring to the PC-MRI measurement data, while those of MR-MI simulation were set to uniform parallel inflow and free stream outflow conditions similar to the UMI simulation.

Figure 2(c) shows PIV-measurement-integrated simulation (PIV-MI simulation) of Karman vortex street behind a square cylinder in a wind tunnel ${ }^{(9)}$. Two-dimensional velocity vector data on a middle plane perpendicular to the cylinder were measured by PIV measurement and fed back to a $2 \mathrm{D}$ flow simulation. The result of $3 \mathrm{D}$ simulation was used as

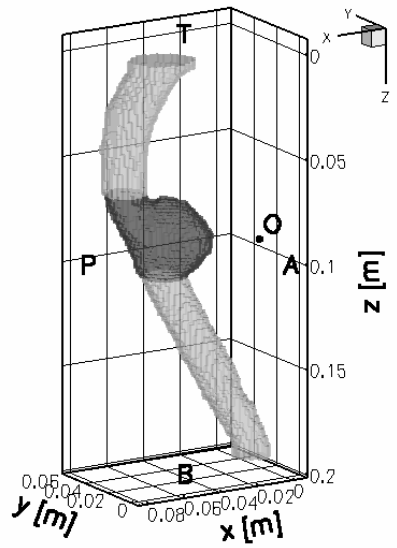

(a) Blood flow in descending aorta with aneurysm (Case 1)

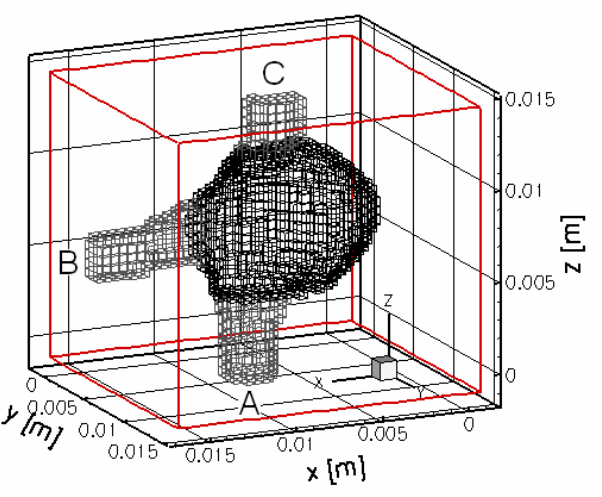

(b) Blood flow in cerebral artery with aneurysm at bifurcation (Case 2)

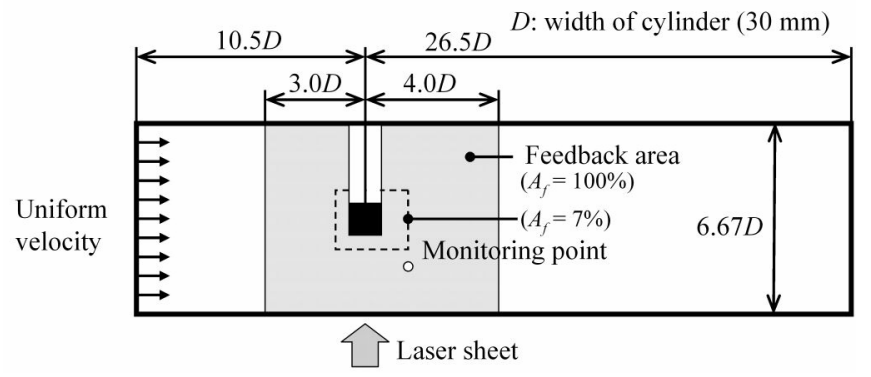

(c) Karman vortex street behind a square cylinder (Case 3)

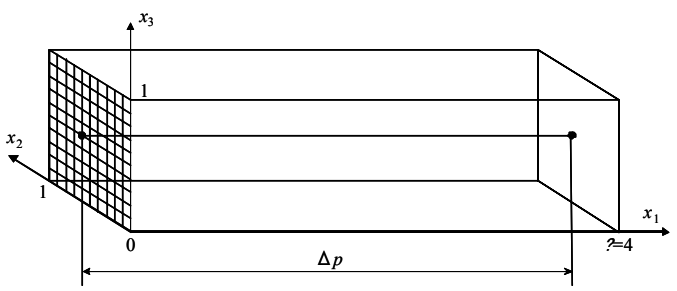

(d) Fully developed turbulent flow in a square duct (Case 4)

Fig. 2 Flow cases treated for verification of theoretical analysis 
the standard solution, and 2D MI simulation was performed with uniform parallel inflow and free stream outflow conditions.

Figure 2 (d) shows MI simulation of a fully developed turbulent flow in a square $\operatorname{duct}^{(7)}$. In this case, we did not specify an actual measurement method and performed a numerical experiment for MI simulation with an ideal measurement with which the complete velocity information is available at all the grid points in a flow domain. As to the boundary condition at the upstream and downstream boundaries, a periodical condition was applied for the velocity, and a constant pressure difference was assumed for the pressure. A sequence of fully developed turbulent flow solutions in a statistically steady state was used as the standard solution, and the velocity field of the standard solution after a sufficient time had passed was used as the initial condition of the MI simulation. All three velocity components at all the grid points in the domain were used for the feedback signal.

In this section, the validity of the approximate sufficient condition of convergence of MI simulation with the first and second order implicit schemes in Eqs. (15) and (21) are examined for Cases 1-4 mentioned above. The validity of the stabilized MI simulation scheme for the first and second order implicit schemes in Eqs. (23) and (25) are then examined for Case 4 of the fully developed turbulent flow in a square duct.

Evaluation of the norm of the feedback gain matrix $\mathbf{K}$ is necessary to examine the approximate sufficient condition of convergence in Eqs. (15) and (21). As mentioned in Eq. (12) in the former section, the norm of $\mathbf{K}$ is defined as the induced norm corresponding to the maximum amplification factor of linear transformation. As to UMI simulation in case 1, in which the external force of the feedback signal is applied in the direction of the ultrasonic beam instead of the velocity error direction, the feedback gain matrix $\mathbf{K}$ is given as the product of a diagonal matrix with a constant diagonal element of the feedback gain $k$ and a matrix representing a projection of a unit vector in the direction of the velocity vector onto the ultrasonic beam direction at all the grid points. From the definition of the norm in Eq. (12), the norm of $\mathbf{K}$ in this case is calculated as $k$. In the other cases in which the external force of the feedback signal is applied to the direction of the velocity error, the feedback gain matrix is a diagonal matrix whose elements are a constant value of $k$. In more general cases in which a feedback signal is applied at partial grid points or for partial velocity components, the corresponding diagonal element of $\mathbf{K}$ is replaced by 0 . In these cases, the norm of $\mathbf{K}$ is also calculated as $k$. Since the norm of the feedback gain matrix $\mathbf{K}$ is identical to the feedback gain $k$ in all the cases treated in this paper, for simplicity, we use the term "feedback gain" also to mean the norm of the feedback gain matrix.

In the numerical experiment, the critical feedback gain for convergence of $\mathrm{MI}$ simulations was determined as follows. In processing MI simulation, the error norm was defined as the average of the absolute value of the velocity vector difference between the MI simulation and the standard solution over the flow domain, and the steady error norm was defined as the steady value of the error norm after the transient period. Convergence of the MI simulation to the standard solution is evaluated using the steady error norm. A number of MI simulations were performed with the feedback gain increased with some increment. The steady error norm first monotonically decreases with increasing feedback gain, but it suddenly increases above some critical gain. The critical feedback gain was determined as the maximum gain just before the sudden increase of the steady error norm.

The same numerical scheme was used for all the cases treated in this paper. Governing equations were discretized with the finite volume method on a equidistant staggered grid

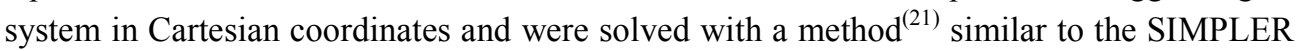
method $^{(16)}$. Convective terms were discretized with the QUICK scheme reformulated considering physical consistency ${ }^{(17)}$, and time derivative terms were discretized with the first or the second order implicit scheme ${ }^{(19)}$. The first order scheme was used in Cases 1a, $2 \mathrm{a}, 2 \mathrm{~b}$, and $4 \mathrm{a}$, and the second order scheme was used in Cases $1 \mathrm{~b}, 3$, and $4 \mathrm{~b}$. 


\subsection{Results and Discussion}

First, we present the results of verification of the approximate sufficient condition of convergence of MI simulation for the first and second order implicit schemes, Eqs. (15) and (21), respectively, for the conditions of the authors' former studies.

Table 2 shows a comparison of critical feedback gain matrix norms (hereafter, abbreviated as critical feedback gains) between the results of the present theory and the numerical experiment for all the cases treated in this study, and Fig. 3 shows the critical feedback gains with the computational time step plotted for the results of the first and second order implicit schemes in Table 2. Critical feedback gains in the numerical experiment were obtained from the literature for UMI simulation in Case $1^{(10)(11)}$ and MR-MI simulation in Case $2^{(12)}$. For PIV-MI simulation in Case 3, the former study adopted the feedback gain of 3 but did not mention about the upper limit of the feedback gain ${ }^{(9)}$. Therefore, calculation was performed here in the same condition as the former study, the feedback gain being increased by an increment of 1 . Since the steady error norm decreased for $k=4$ but increased sharply for $k=5$, the critical feedback gain for this case was determined as 4. As for MI simulation for the turbulent flow in a square duct in Case 4, computation was newly performed to determine the critical feedback gain both for the first and second order implicit schemes. Theoretical values for the critical feedback gain were obtained from Eq. (16) and (22) for the first and second order implicit schemes, respectively.

From Table 2 and Fig. 3, the present theoretical result for the critical feedback gain for the convergence of MI simulation with the first and second order implicit schemes is in good agreement overall with those obtained in the numerical experiment for all the cases treated in this study. Degradation of the agreement for relatively large time steps is ascribed to less satisfaction of the assumption of a small time step and large feedback gain in the present theoretical analysis.

Next, the validity of the proposed stabilized MI simulation scheme is investigated for Case 4 of the turbulent flow in a square duct. MI simulation was performed with the first order implicit schemes of Eqs. (7) and (23) and with the second order implicit schemes of Eqs. (18) and (25). It is noted that the standard solutions in both cases were identical obtained with the second order implicit scheme.

Figure 4 (a) shows the variation of the steady error norm with the feedback gain for the MI simulation of the first order implicit scheme, comparing the proposed scheme (solid line) and the former scheme (broken line). It is noted that the critical feedback gain of convergence of MI simulation obtained from the theoretical analysis is 40 in this case (see Table 2). In the figure, both results are the same for the feedback gain $k \leq 48$. In this range, the steady error norm monotonically decreases with increasing feedback gain. The error

Table 2 Critical feedback gains

\begin{tabular}{|l|c|c|c|c|c|c|c|}
\hline Cases & $1 \mathrm{a}$ & $1 \mathrm{~b}$ & $2 \mathrm{a}$ & $2 \mathrm{~b}$ & 3 & $4 \mathrm{a}$ & $4 \mathrm{~b}$ \\
\hline $\begin{array}{l}\text { Time derivative } \\
\text { discretization }\end{array}$ & $\begin{array}{c}1 \mathrm{st} \\
\text { order }\end{array}$ & $\begin{array}{c}\text { 2nd } \\
\text { order }\end{array}$ & $\begin{array}{c}1 \mathrm{st} \\
\text { order }\end{array}$ & $\begin{array}{c}1 \mathrm{st} \\
\text { order }\end{array}$ & $\begin{array}{c}\text { 2nd } \\
\text { order }\end{array}$ & $\begin{array}{c}1 \mathrm{st} \\
\text { order }\end{array}$ & $\begin{array}{c}\text { 2nd } \\
\text { order }\end{array}$ \\
\hline Time step $\Delta \mathrm{t}$ & $0.01,0.1,1$ & 0.125 & 1.05 & 4.85 & 0.2012 & 0.025 & 0.025 \\
\hline $\begin{array}{l}\text { Critical feedback } \\
\text { gain matrix norm } \\
\text { (Theory) }\end{array}$ & $100,10,1$ & 4 & 0.95 & 0.21 & 2.5 & 40 & 20 \\
\hline $\begin{array}{l}\text { Critical feedback } \\
\text { gain matrix norm } \\
\text { (Numerical } \\
\text { experiment) }\end{array}$ & $160,20,4$ & 11 & 3.5 & 2 & 4 & 48 & 16 \\
\hline
\end{tabular}


norm with the former scheme suddenly increases in the range of the feedback gain larger than 64 due to the destabilization phenomenon, but that of the proposed scheme is continuously decreasing in this region, with no occurrence of the destabilization phenomenon.

Figure 4 (b) shows a comparison between the proposed scheme (solid line) and the former scheme (broken line) for the results of the second order implicit scheme. The critical feedback gain obtained from the theoretical analysis is 20 in this case (see Table 2). In the figure, both results are nearly the same for the feedback gain $k \leq 16$. In this range the steady error norm monotonically decreases and then remains at almost the same level with increasing feedback gain. The error norm with the former scheme suddenly increases in the range of the feedback gain larger than 32 due to the destabilization phenomenon, but that of the proposed scheme remains at a small level, showing that no destabilization phenomenon occurs. It is also noted that the first reduction of the steady error norm with increasing

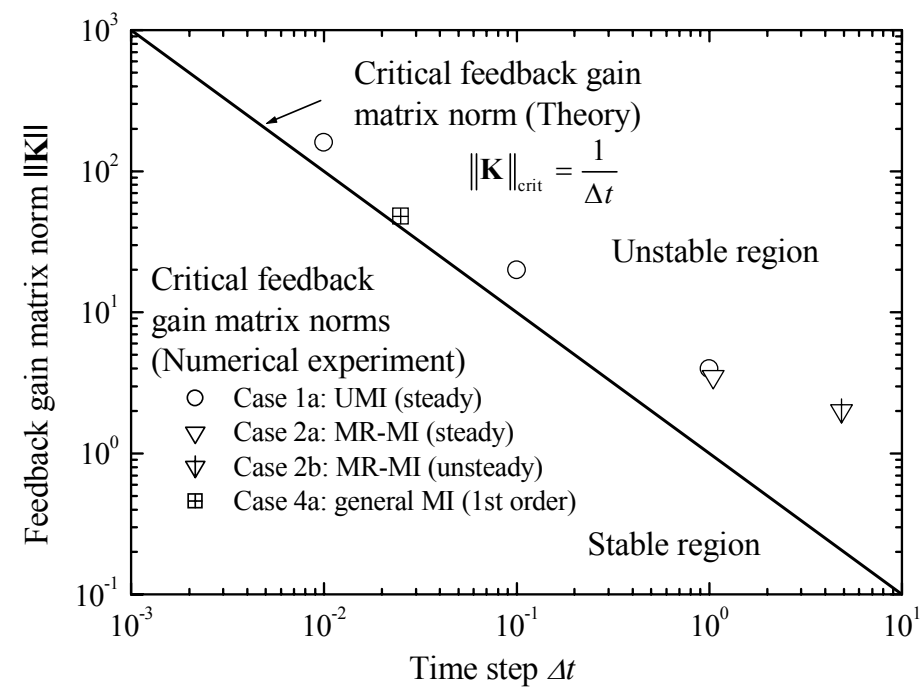

(a) 1st order scheme

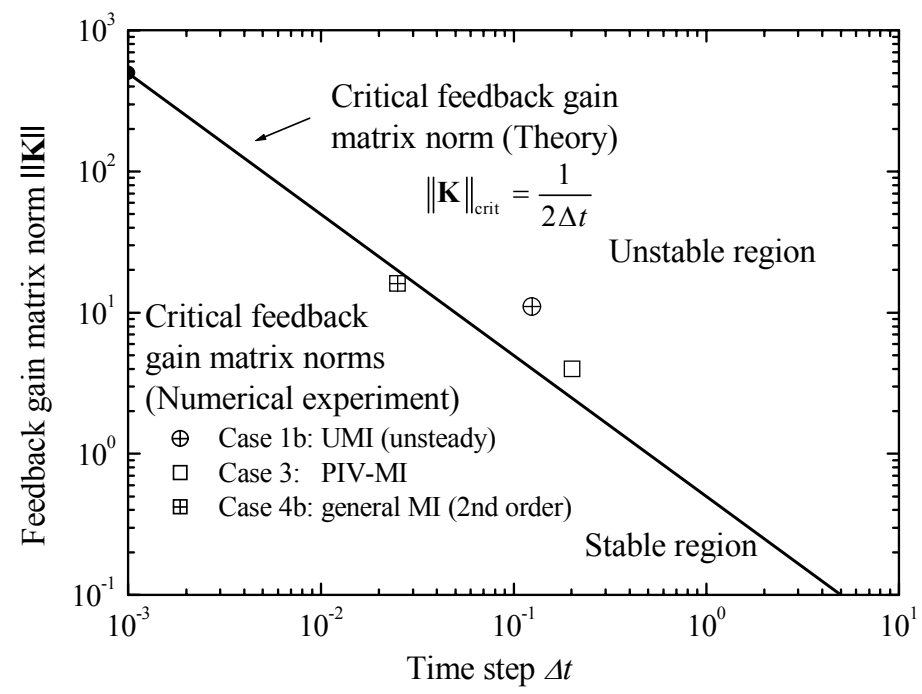

(b) 2nd order scheme

Fig. 3 Critical feedback gain matrix norms with the computational time step compared between the present theory and numerical experiment for various flow cases. 
feedback gain is more significant for the case of the second order scheme of Fig. 4 (b) in comparison with that of the first order scheme of Fig. 4 (a). This is because the second order implicit scheme was used to obtain the standard solutions in both cases.

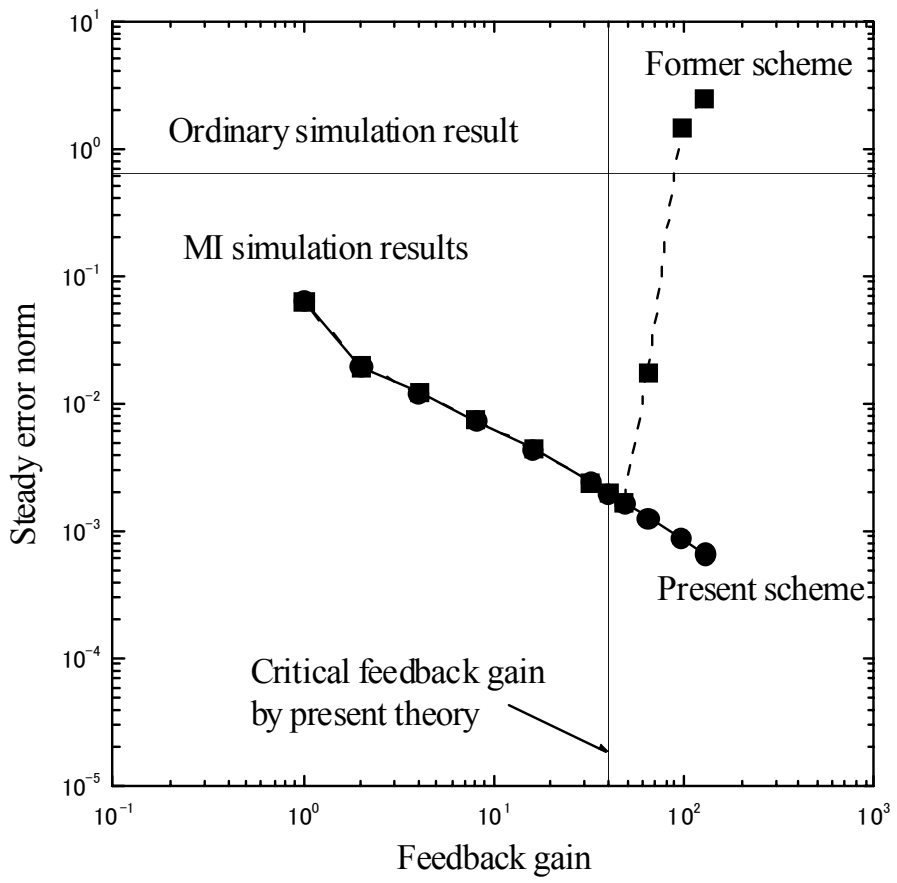

(a) 1 st order scheme (Case 4a)

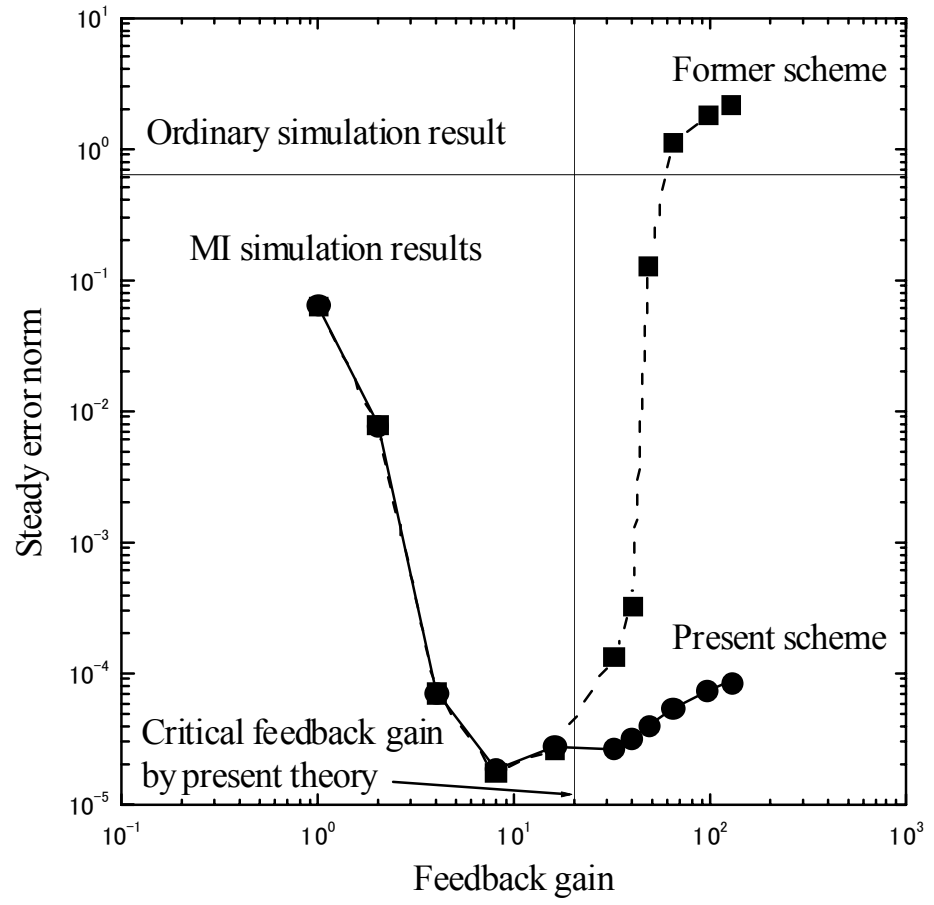

(b) 2nd order scheme (Case $4 b)$

Fig. 4 Comparison between the former MI simulation scheme and the present stabilized scheme for the variation of the steady error norm with the feedback gain. Fully developed turbulent flow in a square duct (Case 4) 


\section{Conclusions}

In this paper we have theoretically dealt with the destabilization phenomenon of MI simulation, in which the estimation error suddenly increases with a feedback gain above some critical value. This phenomenon has been considered as instability of a closed-loop feedback system, but present study treated it as that of a numerical scheme. First, the mechanism of destabilization phenomenon was investigated based on the sufficient condition of the convergence of iterative calculation of existing MI simulation. It was found that the feedback signal in the source term destabilized the iterative calculation. Then, a new MI simulation scheme was derived by evaluating the feedback signal in the linear term to remove the cause of the destabilization. The validity of the theoretical analysis was verified for examples of MI simulation for the ultrasonic-measurement-integrated simulation of blood flow in an aneurismal aorta, MR-measurement-integrated simulation of blood flow in a cerebral aneurism, PIV-measurement-integrated simulation of Karman vortex street behind a square cylinder, and general MI simulation of fully developed turbulent flow in a square duct. Occurrences of the destabilization phenomenon in all examples were well explained by the approximate sufficient condition of the convergence of MI simulation, especially for cases with relatively small time steps and large feedback gains. Furthermore, the new MI simulation schemes with the first and second order implicit schemes realized the stable MI simulation without the destabilization phenomenon for large feedback gains. The theoretical result of this paper confirming that the destabilization phenomenon is not the instability of the feedback system but that of the numerical scheme is generally applicable to MI simulations using the velocity error in determining the feedback signal. A natural extension to other cases of MI simulations using the pressure error to determine the feedback signal is a future work.

\section{Acknowledgements}

Part of this study was supported by JST System Development Program for Advanced Measurement and Analysis (Program-S), Grant-in-Aid for Scientific Research (B) (19360080), the Tohoku University Global COE Program "Global Nano-Biomedical Engineering Education and Research Network Centre". Computation in this study was performed at the Advanced Fluid Information Research Center, Institute of Fluid Science, Tohoku University.

\section{References}

(1) Munro, R., Kopken, C., Kelly, G., Thepaut, J.N., Saunders, R., Assimilation of Meteosat radiance data within the 4D-Var system at ECMWF: Data quality monitoring, bias correction and single-cycle experiments. Quarterly Journal of the Royal Meteorological Society. Vol. 130, No. 601(2004), pp. 2293-2313.

(2) Szunyogh, I., Kostelich, E.J., Gyarmati, G., Patil, D.J., Hunt, B.R., Kalnay, E., Ott, E., Yorke, J.A., Assessing a local ensemble Kalman filter: perfect model experiments with the National Centers for Environmental Prediction global model. Tellus Series a-Dynamic Meteorology and Oceanography. Vol. 57, No. 4(2005), pp. 528-545.

(3) Zeldin, B.A., Meade, A.J., Integrating experimental data and mathematical models in simulation of physical systems. AIAA Journal. Vol. 35, No. 11(1997), pp. 1787-1790.

(4) Ido, T., Murai, Y., Yamamoto, F., Postprocessing algorithm for particle-tracking velocimetry based on ellipsoidal equations. Experiments in Fluids. Vol. 32, No. 3(2002), pp. 326-336.

(5) Uchiyama, M., Hakomori, K., Measurement of Instantaneous Flow-Rate through Estimation of Velocity Profiles. IEEE Transactions on Automatic Control. Vol. 28, No. 3(1983), pp. 380-388.

(6) Hayase, T., Hayashi, S., State estimator of flow as an integrated computational method with the feedback of online experimental measurement. Journal of Fluids Engineering-Transactions of the ASME. Vol. 119, No. 4(1997), pp. 814-822. 
(7) Imagawa, K., Hayase, T., Numerical Experiment of Measurement-Integrated Simulation to Reproduce Turbulent Flows with Feedback Loop to Dynamically Compensate the Solution Using Real Flow Information. Computers and Fluids. Vol. 39, No. 9 (2010), pp. 1439-1450.

(8) Nisugi, K., Hayase, T., Shirai, A., Fundamental study of hybrid wind tunnel integrating numerical simulation and experiment in analysis of flow field. JSME International Journal Series B-Fluids and Thermal Engineering. Vol. 47, No. 3(2004), pp. 593-604.

(9) Yamagata, T., Hayase, T., Higuchi, H., Effect of Feedback Data Rate in PIV Measurement-Integrated Simulation. Journal of Fluid Science and Technology. Vol. 3, No. 4(2008), pp. 477-487.

(10) Funamoto, K., Hayase, T., Saijo, Y., Yambe, T., Numerical experiment for Ultrasonic-Measurement-Integrated simulation of three-dimensional unsteady blood flow. Annals of Biomedical Engineering. Vol. 36, No. 8(2008), pp. 1383-1397.

(11) Funamoto, K., Hayase, T., Saijo, Y., Yambe, T., Numerical Experiment of Transient and Steady Characteristics of Ultrasonic-Measurement-Integrated Simulation in Three-Dimensional Blood Flow Analysis. Annals of Biomedical Engineering. Vol. 37, No. 1(2009), pp. 34-49.

(12) Funamoto, K., Suzuki, Y., Hayase, T., Kosugi, T., Isoda, H., Numerical Validation of MR-Measurement-Integrated Simulation of Blood Flow in a Cerebral Aneurysm. Annals of Biomedical Engineering. Vol. 37, No. 6(2009), pp. 1105-1116.

(13) Nakao, M., Kawashima, K., Kagawa, T., Application of MI Simulation Using a Turbulent Model for Unsteady Orifice Flow. Journal of Fluids Engineering-Transactions of the ASME. Vol. 131, No. 11(2009), pp. 111401-1-111401-6.

(14) Imagawa, K., Hayase, T., Eigenvalue analysis of linearized error dynamics of measurement integrated flow simulation. Computers and Fluids. Vol. 39, No. 10 (2010), pp. 1796-1803.

(15) Dorf, R.C., Modern Control Systems. (1967), 135, Addison-Wesley.

(16) Patankar, S.V., Numerical heat transfer and fluid flow (1980), Hemisphere Pub. Corp.

(17) Hayase, T., Humphrey, J.A.C., Greif, R., A Consistently Formulated Quick Scheme for Fast and Stable Convergence Using Finite-Volume Iterative Calculation Procedures. Journal of Computational Physics. Vol. 98, No. 1(1992), pp. 108-118.

(18) Watkins, D.S., Fundamentals of matrix computations. (1991), 91, Wiley, New York.

(19) Fletcher, C.A.J., Computational Techniques for Fluid Dynamics.Vol.1. (1988), 302, Springer-Verlag.

(20) Biomedical Engineering Handbook, ed. J. Bronzino. (2000), CRC Press.

(21) Hayase, T., Humphrey, J.A.C., Greif, R., Mini Manual for ROTFLO2. Department of Mechanical Engineering Report, University of California at Berkeley. FM-90-1, (1990). 